\title{
Notes on Anderson-Anderson-Johnson questions and Bouvier questions
}

\author{
RYûKI MatsudA*
}

\section{$\S 0$. Introduction.}

In this paper $A$ denotes an integral domain. A domain $A$ is called a locally factorial ring if $A_{M}$ is a factorial ring for each maximal ideal $M$ of $A$. If each ideal of $A$ is a product of primary ideals of $A$, then $A$ is called a Q-ring.

Let $X^{(1)}(A)$ be the set of height one prime ideals of $A$, and $\operatorname{Spec}(A)$ the set of prime ideals of $A$.

$[6, \mathrm{p} .17]$ asks the following question : ring?

(B-0) Let $A$ be a locally factorial ring with $\operatorname{Pic}(A)=0$. Is $A$ a factorial

Let $A$ be a Krull ring. $[3, \S 2]$ asks the following questions :

Are the following statements true?

(AAJ-1) Let $P$ be a height one prime ideal of $A$. If $P \cap Q=P Q$ for each $Q \in X^{(1)}(A)$ with $Q \neq P$, then $P$ is an invertible ideal.

(AAJ-2) Let $P$ be a non-zero prime ideal of $A$. If $P \cap Q=P Q$ for each $Q \in \operatorname{Spec}(A)$ with $h t Q \leqq 2$ that is incomparable with $P$, then $P$ is a maximal ideal or an invertible ideal of $A$.

(AAJ-3) $A$ is a locally factorial ring if and only if $P \cap Q=P Q$ for each pair of distinct height one prime ideals $P, Q$ of $A$.

(AAJ-4) $A$ is a factorial ring if and only if $P i c(A)=0$ and $P \cap Q=P Q$ for each pair of distinct height one prime ideals $P, Q$ of $A$.

(AAJ-5) $A$ is a locally factorial ring if and only if $P Q$ is a divisorial ideal for each distinct height one prime ideals $P, Q$ of $A$.

(AAJ-6) The following conditions are equivalent:

(1) $A$ is a locally factorial ring with $\operatorname{dim} A \leqq 2$.

(2) $P \cap Q=P Q$ for each incomparable prime ideals $P, Q$ of $A$.

(3) $\mathrm{A}$ is a $\mathrm{Q}$-ring.

Let $A$ be a Krull ring. Relating with the above questions, $[6,(3.9)]$ asks the following questions;

(B-1) Are the following conditions equivalent?

(1) $A$ is a locally factorial ring.

(2) $P \cap Q=P Q$ for each distinct height one prime ideals $P, Q$ of $A$.

(3) $P Q$ is a divisorial ideal for each height one prime ideals $P, Q$ of $A$.

(B-2) If $\operatorname{Pic}(A)=0$ and $P \cap Q=P Q$ for each distinct height one prime ideals $P, Q$ of $A$, then is $A$ a factorial ring?

Received March 25, 1989.

* Department of Mathematics, Ibaraki University, Mito, Ibaraki 310, Japan. 
(B-3) Let $A$ be a locally factorial domain. Then are the following conditions equivalent?

(1) $\operatorname{dim} A \leqq 2$.

(2) $P \cap Q=P Q$ for each incomparable prime ideals $P, Q$ of $A$.

(3) $A$ is a $Q$-ring.

§1. On (B-0).

(1.1). Let $Z$ be the ring of integers, $\left\{p_{i} ; i=1,2, \cdots\right\}$ the set of positive prime numbers, and $X, Y$ indeterminates over $Z$. Set $A=$

$\bigcup_{i=1}^{\infty} \mathrm{Z}\left[X / p_{1}, Y / p_{1}, X / p_{2}, Y / p_{2}, \cdots, X / p_{n}, Y / p_{n}\right]$ (cf. [5, Example]). Then we have the following,

(a) $A$ is not a Krull domain. Thus $A$ is not a factorial ring.

(b) $A$ is a locally factorial ring with $\operatorname{Pic}(A)=0$.

Proof. (a): [5,Example,(a)] shows that $A$ is not a Krull domain.

(b): Let $\mathbf{Q}$ be the field of rational numbers, and $M$ be a maximal ideal of $A$. Then $A_{M}$ is a localization of $\mathrm{Q}[X, Y]$ or $A_{M}$ is a localization of the polynomial ring $\mathbf{Z}_{(p)}[X / p, Y / p]$ for a prime number $p$. Hence $A_{M}$ is a factorial ring. Thus $A$ is a locally factorial ring. Set $A_{n}=\mathrm{Z}\left[X /\left(p_{1} \cdots p_{n}\right), Y /\left(p_{1} \cdots p_{n}\right)\right]$ for each natural number $n$. We have $A_{1} \subseteq A_{2} \subseteq \cdots \subseteq A_{n} \subseteq \cdots$, and $A=\bigcup_{i=1}^{\infty} A_{n}$. Thus $\operatorname{Pic}(A)=\lim \left\{\operatorname{Pic}\left(A_{n}\right)\right\}$ by [8,Theorem 1.3]. Since each $A_{n}$ is a factorial ring, we have $\operatorname{Pic}(A)=0$.

Thus Question (B-0) was solved negatively.

\section{§2. Lemmas.}

(2.1)([2, Corollary 2.2]). Let $A$ be a Krull ring, $I$ a nonzero ideal of $A$. Then $I$ is an invertible ideal of $A$ if and only if $I$ is a locally principal ideal of $A$. Q-ring.

(2.2)(A PART of [4]). (1) If $A$ is a Q-ring, then any quotient ring is a

(2) Let $(A, M)$ be a quasi-local ring. Then $A$ is a $\mathrm{Q}$-ring if and only if $A$ is either $\operatorname{dim} A \leqq 1$ or $A$ is a 2 -dimensinal factorial ring.

(3) $A$ is a $Q$-ring if and only if $A$ is Laskerian and every nonmaximal prime ideal is finitely generated and locally principal.

(2.3)(A PART of [1, TheOREM 1]). Let $A$ be a Krull ring. Then the followings are equivalent:

(1) $A$ is a locally factorial ring.

(2) Each height one prime ideal of $A$ is an invertible ideal.

(3) The product of two divisorial ideals of $A$ is a divisorial ideal. 


\section{§3. The implications of the questions.}

(3.1).

(1) Let $P, Q \in \operatorname{Spec}(A)$. Then $P \cap Q=P Q$ if and only if $P A_{M} \cap Q A_{M}=P A_{M} \cdot Q A_{M}$ for each $M \in \operatorname{Max}(A)$.

(2) Let $P \in \operatorname{Spec}(A)$ and let $P \subset M \in \operatorname{Max}(A)$. Then $P \supsetneqq P M$ if and only if $P A_{M} \supsetneqq P A_{M} \cdot M A_{M}$.

(3.2). Let $A$ be a Krull ring and let $P, Q \in X^{(1)}(A)$ with $P \neq Q$. Then

(1) $P Q$ is a divisorial ideal of $A$ if and only if $P \cap Q=P Q$.

(2) If $P Q$ is a divisorial ideal of $A$, then $P A_{M} \cdot Q A_{M}$ is a divisorial ideal of $A_{M}$ for each $M \in \operatorname{Max}(A)$.

Proof. (1): Assume that $P Q$ is a divisorial ideal. We have $\operatorname{div}(P \cap Q)=\sup (\operatorname{div} P, \operatorname{div} Q)=\operatorname{div} P+\operatorname{div} Q=\operatorname{div} P Q$.

Thus $P \cap Q=(P Q)^{v}=P Q$.

(2) follows by (1).

(3.3).

(1) In (AAJ-3), the necessity holds.

(2) In (AAJ-4), the necessity holds.

(3) In (AAJ-5), the necessity holds.

(4) In (AAJ-6); (3) implies (1), and (1) implies (2).

(5) In (B-1); (1) implies (3), and (3) implies (2).

(6) In (B-3); (3) implies (1), and (1) implies (2).

Proof. (1) and (3) follow by (2. 3).

(4) and (6) follow by (2. 2).

(5): (1) implies (3) by (2. 3). (3) implies (2) by (3. 2).

Let (A) be a 'yes or no' question for domains. Assume that if $(A)$ is yes for each quasi-local domain, then (A) is yes for each domain. Then we will say that (A) is of local property.

(3.4).

(1) (AAJ-1) is of local property.

(2) (AAJ-2) is of local property.

(3) (AAJ-3) is of local property.

(4) (AAJ-4) is of local property.

(5) (AAJ-5) is of local property.

(6) (B-1) is of local property.

(7) (B-2) is of local property.

Proof. (1) and (2) follow by (2. 1).

(4): Let $A$ be a Krull ring. We will show the sufficiency in (AAJ-4). By assumption, $A$ is a locally factorial ring. By (2. 3), each height one prime ideal of $A$ is an invertible ideal. Since $\operatorname{Pic}(A)=0, A$ is a factorial ring.

(5) follows by (3. 2).

(3.5). If (AAJ-2) is yes, then (AAJ-1) is yes. 
Proof. Let $(A, M)$ be a quasi-local Krull domain and let $P \in X^{(1)}(A)$. Assume that $P \cap Q=P Q$ for each $Q \in X^{(1)}(A)$ with $Q \neq P$. We will show that $P$ is an invertible ideal.

If $\operatorname{dim} A \leqq 1$, then $P$ is an invertible ideal.

Let $\operatorname{dim} A \geqq 2$ and let $Q \in \operatorname{Spec}(A)$ with $h t Q \leqq 2$ that is incomparable with $P$. If $h t Q \leqq 1$, then $P \cap Q=P Q$. Suppose that $h t Q=2$. Take $a \in P \cap Q$ with $a \neq 0$. Since $A_{Q}$ is a Krull domain and $a \in Q A_{Q}$, there exists $Q^{\prime} \in X^{(1)}\left(A_{Q}\right)$ such that $a \in Q^{\prime}$. $Q^{\prime}$ is of the form $Q_{0} A_{Q}$ where $Q \supsetneqq Q_{0} \in X^{(1)}(A), P \neq Q_{0}$ and $a \in P \cap Q_{0}=P Q_{0} \subset P Q$. Thus $P \cap Q=P Q$. Since (AAJ-2) is yes, $P$ is a maximal ideal or an invertible ideal. Since $\operatorname{dim} A \geqq 2, P$ is an invertible ideal of A.

Let $\left(\mathrm{A}^{\prime}\right)$ and $\left(\mathrm{A}^{\prime \prime}\right)$ be two 'yes or no' questions. In the followings ' $\left(A^{\prime}\right) \Longrightarrow$ $\left(A^{\prime \prime}\right)^{\prime}$ means that if $\left(\mathrm{A}^{\prime}\right)$ is yes then $\left(\mathrm{A}^{\prime \prime}\right)$ is yes.

(3.6).

(1) $(A A J-2) \Longrightarrow(A A J-1) \Longrightarrow(A A J-3) \Longleftrightarrow(A A J-4)$

$\Longleftrightarrow(A A J-5) \Longleftrightarrow(B-1) \Longleftrightarrow(B-2)$.

(2) $(A A J-6) \Longrightarrow(B-3)$.

(3.7). If $(A A J-2)$ is yes, then we have the followings:

(1) In (AAJ-6); (1) $\Longleftrightarrow$ (2).

(2) In (B-3); (1)

(3) $(A A J-6) \Longleftrightarrow(B-3)$.

(3.8). Let $A$ be a Krull ring and let $P \in X^{(1)}(A)$. Then $P$ is an invertible ideal of $A$ if and only if $P \cap Q=P Q$ for each $P \neq Q \in X^{(1)}(A)$ and $P \nsupseteq P M$ for each $P \subset M \in \operatorname{Max}(A)$.

Proof. The sufficiency: We may assume that $(\mathrm{A}, \mathrm{M})$ is a quasi-local ring. Let $P \in X^{(1)}(A)$. Since $A$ is a Krull ring, $A$ is an atomic ring. Take $a \in P-P M$. Then $a$ is an irreducible element of $A$. Because, if $a$ is a reducible element with $a=p_{1} p_{2} \cdots p_{n}$, where $n \geqq 2$ and each $p_{i}$ is irreducible element, then $p_{i} \in P$ for some $i$. Thus $a \in P M$; a contradiction.

Let $Q \in X^{(1)}(A)$ that is distinct with $P$. If $a \in Q$, then $a \in P \cap Q=P Q \subseteq$ $P M$; a contradiction. Thus $P$ is the only height one prime ideal containing $a$.

Let $v$ be the valuation with the valuation ring $A_{P}$. Then we have $v(a)=v(b)$ for each $b \in P-P M$. Because, if , say, $v(a) \lessgtr v(b)$ then there exists $x$ of $A$ such that $x=b / a$. Then $b=a x \in P M$; a contradiction. Thus $P=a A \cup P M$. It follows that $P=a A$.

\section{§4. The integral closure of a Noetherian domain.}

A Noetherian ring $A$ is called a local ring if $A$ has only one maximal ideal. Let $B$ be a Noetherian domain with quotient field $L, K$ a finite algebraic extention field of $L$ and $A$ the integral closure of $B$ in $K$. By Mori-Nagata's Integral Closure Theorem, $A$ is a Krull ring. 
(4.1)(Goro). Let $A$ be as above. Then we have $\bigcap_{n=1}^{\infty} P^{n} A_{P}=(0)$ for each $P \in \operatorname{Spec}(A)$.

Proof. Set $P \cap B=P^{\prime}$. Then $A_{P^{\prime}}$ is the integral closure of $B_{P^{\prime}}$ in $K$. Thus we may assume that $(B, M)$ is a local ring and $P \cap B=M$.

Set $d=\operatorname{dim} B$. Thus we may use the induction on $d$.

If $d=0,1$, then the statement is clear. Suppose that $d \geqq 2$ and the statement holds for all positive integer lower than $d$. Set $n=[\bar{K}: L]$. Then $K=\sum_{i=1}^{n} L x_{i}$ for some $x_{1}, x_{2}, \cdots, x_{n} \in A$. Set $M^{\prime}=P \cap B\left[x_{1}, \cdots, x_{n}\right]$. We may replace $B$ with $B\left[x_{1}, x_{2}, \cdots, x_{n}\right]_{M}$ and $K$ with $L$.

Then $A$ is the integral closure of $B$ in $K$.

We may assume that $h t P \geqq 2$. Take $Q \in X^{(1)}(A)$ with $Q \subset P$. Set $Q \cap B=Q^{\prime}$. Then $Q^{\prime} \neq(0), B / Q^{\prime} \subset A / Q$ and the quotient field of $A / Q$ is a finite algebraic extension of that of $B / Q^{\prime}$ by Mori-Nagata's Theorem. Let $R$ be the integral closure of $B / Q^{\prime}$ in the quotient field of $A / Q$. We have $\operatorname{dim}\left(B / Q^{\prime}\right)<d$. Take $N \in \operatorname{Spec}(R)$ with $N \cap(A / Q)=P / Q$. Since $\operatorname{dim} R<d$, we have

(0) $=\bigcap_{n=1}^{\infty} N^{n} R_{N} \supset \bigcap_{n=1}^{\infty} P^{n}\left(A_{P} / Q A_{P}\right)$.

Thus

$\bigcap_{n=1}^{\infty} P^{n} A_{P} \subset \bigcap_{Q \in X_{Q \subset P}^{(1)}(A)} Q A_{P}$

Since $\operatorname{dim} A_{P} \geqq 2$, it follows that $\bigcap_{Q \in X_{Q \subset P}^{(1)}(A)} Q A_{P}=(0)$.

Thus $\bigcap_{n=1}^{\infty} P^{n} A_{P}=(0)$.

(4.2). Let $B$ be a Noetherian domain with quotient field $L$. Let $K$ be a finite algebraic extension field of $L$, and $A$ the integral closure of $B$ in $K$. then $(A A J-1)$ is yes for $A$.

Proof. Let $P \in X^{(1)}(A)$. Assume that $P \cap Q=P Q$ for each $Q \in X^{(1)}(A)$ with $P \neq Q$. Let $P \subset M \in M a x(A)$. If $P=P M$, then

$P A_{M}=P M A_{M}=P M^{2} A_{M}=P M^{3} A_{M}=\cdots=(0)$

by (4. 1); a contradiction. Therefore $P \supsetneqq P M$. (3.8) implies that $P$ is an invertible ideal.

(4.3). Let $A$ be as (4.2). Then for $A$ we have

$(A A J-6) \Longleftrightarrow(B-3)$.

Proof. The sufficiency: we assume (2) of (AAJ-6). We will prove (3) of (AAJ-6). Each height one prime ideal of $A$ is an invertible ideal by (4.2). Therefore $A$ is a locally factorial ring. Since (B-3) is yes, $A$ is a Q-ring.

(4.4). Let $A$ be as (4. 2). Then for $A$ we have $(A A J-2) \Longrightarrow(B-3)$.

Proof. We assume moreover that $A$ is locally factorial. Assume (2) of (B-3). We will show (3) of (B-3). Since (AAJ-2) is yes for $A$, we have $\operatorname{dim} A \leqq 2$. By Nagata's Theorem, $A$ is a Noetherian ring. By [3, Corollary (2. 8)], $A$ is a Q-ring. 


\section{§5. An example}

Relating with (4.1), there naturaly arises the following question:

Let $(A, M)$ be a quasi-local Krull domain. Then is $\bigcap_{n=1}^{\infty} M^{n}=(0)$ ? If the answer to this question is yes, then (AAJ-1) is yes.

(5.1). There exists a quasi-local factorial ring (A,M) with $M \neq(0)$ such that $\bigcap_{n=1}^{\infty} M^{n}=M$.

In fact, let $A$ be a factorial ring, $X, U_{1}, X_{1}, Y_{1}$ be indeterminates. Set $V_{1}=\left(X-U_{1} X_{1}\right) / Y_{1}, D^{\prime}=A\left[U_{1}, X_{1}, V_{1}, Y_{1}\right]$ and $D=A[X]$. Then we have the following,

(1) $h t\left(P^{\prime} \cap D\right) \leqq 1$ for each $P^{\prime} \in X^{(1)}\left(D^{\prime}\right)$.

(2) For each $P \in X^{(1)}(D)$ there exists a unique $P^{\prime} \in X^{(1)}\left(D^{\prime}\right)$ such that $P^{\prime} \cap D=P$. Further $P^{\prime} D_{P^{\prime}}^{\prime}=P D_{P^{\prime}}^{\prime}$.

Let $k$ be a field and let $\left\{X\left(i_{1} i_{2} \cdots i_{n}\right) ; n, i_{j} \in \mathrm{N}, 1 \leqq i_{j} \leqq 4\right\}$ be a system of indeterminates. Set

$Y\left(i_{1} \cdots i_{n}\right)=X\left(i_{1} \cdots i_{n}\right)-X\left(i_{1} \cdots i_{n} 1\right) X\left(i_{1} \cdots i_{n} 2\right)$

$-X\left(i_{1} \cdots i_{n} 3\right) X\left(i_{1} \cdots i_{n} 4\right)$.

Let $k\left[X\left(i_{1} \cdots i_{n}\right) ; i_{1}, \cdots, i_{n}\right]$ be a polynomial ring over $k$ of the infinite number of indeterminates. Let $\left(Y\left(i_{1} \cdots i_{n}\right) ; i_{1}, \cdots, i_{n}\right)$ be the ideal of

$k\left[X\left(i_{1}, \cdots, i_{n}\right) ; i_{1}, \cdots, i_{n}\right]$ generated by the elements

$\left\{Y\left(i_{1} \cdots i_{n}\right) ; i_{1}, \cdots, i_{n}\right\}$. Then

$\left(Y\left(i_{1} \cdots i_{n}\right) ; i_{1}, \cdots, i_{n}\right)$ is a prime ideal of $k\left[X\left(i_{1} \cdots i_{n}\right) ; i_{1}, \cdots, i_{n}\right]$. Set

$D=k\left[X\left(i_{1} \cdots i_{n}\right) ; i_{1}, \cdots, i_{n}\right] /\left(Y\left(i_{1} \cdots i_{n}\right) ; i_{1}, \cdots, i_{n}\right)$. Let $x\left(i_{1} \cdots i_{n}\right)$ be the image of $X\left(i_{1} \cdots i_{n}\right)$ under the natural homomorphism of

$k\left[X\left(i_{1} \cdots i_{n}\right) ; i_{1}, \cdots, i_{n}\right]$ to $D$. Then we have $D=\bigcup_{i=1}^{\infty} k\left[x\left(i_{1} \cdots i_{n}\right) ; n \leqq\right.$ $\left.i, 1 \leqq i_{j} \leqq 4\right]$, which we denote by $k\left[x\left(i_{1} \cdots i_{n}\right) ; i_{1}, \cdots, i_{n}\right]$. We have

$x\left(i_{1} \cdots i_{n}\right)=x\left(i_{1} \cdots i_{n} 1\right) x\left(i_{1} \cdots i_{n} 2\right)+x\left(i_{1} \cdots i_{n} 3\right) x\left(i_{1} \cdots i_{n} 4\right)$.

Set

$D_{0}=k[x(1), x(2), x(3), x(4)]$,

$D_{1}=D_{0}[x(11), x(12), x(13), x(14)]$,

$D_{2}=D_{1}[x(21), x(22), x(23), x(24)]$,

$D_{3}=D_{2}[x(31), x(32), x(33), x(34)]$,

$D_{4}=D_{3}[x(41), x(42), x(43), x(44)]$,

$D_{5}=D_{4}[x(111), x(112), x(113), x(114)]$,

$D_{6}=D_{5}[x(121), x(122), x(123), x(124)]$,

Then we have $D=\bigcup_{i=1}^{\infty} D_{i}$.

Each $D_{i}$ is a polynomial ring over $k$. Hence each $D_{i}$ is a factorial ring. For each $n \in \mathbf{N}, D_{n}$ and $D_{n+1}$ are of the following forms:

$D_{n+1}=A[x(\alpha 1), x(\alpha 2), x(\alpha 3), x(\alpha 4)]$,

$D_{n}=A[x(\alpha)]$, where $A$ is some factorial ring and

$x(\alpha)=x(\alpha 1) x(\alpha 2)+x(\alpha 3) x(\alpha 4)$. Thus $h t\left(P^{\prime} \cap D_{n}\right) \leqq 1$ for each $P^{\prime} \in$ $X^{(1)}\left(D_{n+1}\right)$ by $(1)$. 
Let $m \gtreqless n$ and let $P_{m}$ be a height one prime ideal of $D_{m}$ such that $P_{m} \cap D_{n}=$ $P_{n}$ for some $P_{n} \in X^{(1)}\left(D_{n}\right)$. Then we have $P_{n}\left(D_{m}\right)_{P_{m}}=P_{m}\left(D_{m}\right)_{P_{m}}$ by (2). Thus $P_{n} \not \subset\left(P_{m}\right)^{(2)}$ (the 2-nd symbolic power of $\left.P_{m}\right)$. Thus $D$ is a Krull domain by $\left[7\right.$,Theorem 3]. By [7, Remark 2] we have $X^{(1)}(D)=\bigcup_{n=1}^{\infty} X^{(1)}\left(D_{n}\right)$. Hence $D$ is a factorial domain. Set $N=\left(x\left(i_{1} \cdots i_{n}\right) ; i_{1}, \cdots, i_{n}\right) . N$ is a maximal ideal of $D$. We have $N=N^{2}=N^{3}=\cdots$. Set $A=D_{N}$. Then $A$ is a quasi-local factorial domain with maximal ideal $M=N D_{N}$. Moreover we have $\bigcap_{n=1}^{\infty} M^{n}=M$.

\section{References}

[1] D.D.Anderson, $\pi$-domains,overrings,and divisorial ideals, Glasgow Math.J., 19 (1978), 199-203.

[2] D.D.Anderson, Globalization of some local properties in Krull domains, Proc.Amer. Math.Soc., 85 (1982), 141-145.

[3] D.D.Anderson,D.F.Anderson and E.W.Johnson, Some ideal-theoretic conditions on a Noetherian ring, Houston J.Math., 7 (1981), 1-10.

[4] D.D.Anderson and L.A.Mahaney, Commutative rings in which every ideal is a product of primary ideals, J.Alg., 106 (1987), 528-535.

[5] J.T.Arnold and R.Matsuda, An almost Krull domain with divisorial height one primes, Canad.Math.Bull., 29 (1986), 50-53.

[6] A.Bouvier, Survey of Locally Factorial Krull Domains, Queen's Math.,Queen's Univ., 1980.

[7] R.Gilmer and W.Heinzer, Rings of formal power series over a Krull domain, Math.Z., 106 (1968), 379-387.

[8] R.Gilmer and R.Heitmann, On Pic(R[X]) for R seminormal, J.Pure and Applied Alg., 16 (1980), 251-257. 